\title{
COMPUTATION OF CHANGES IN LOCAL QUANTITIES OF INTEREST DUE TO DESIGN MODIFICATIONS
}

\author{
Daniel Materna \\ Department of Civil Engineering \\ Ostwestfalen-Lippe University of Applied Sciences and Arts \\ Emilienstraße 45, 32756 Detmold, Germany \\ daniel.materna@th-owl.de
}

Key words: Quantities of interest, design modifications, reanalysis, primal and dual problems

\begin{abstract}
In this contribution a method for the prediction of the change in a quantity of interest due to structural modifications is considered. The approach is based on a goal-oriented method by using the primal and dual problems. The method can be used in order to reduce the overall computational cost in the numerical simulation. Furthermore, the proposed method is easily to implement in existing finite element programs, because no derivatives with respect to the design variables are necessary.
\end{abstract}

\section{Introduction}

Many problems deal with structural analysis but also with the investigation of the behavior due to given design modifications (design changes), e.g. the shape, the topology, material properties etc. Classical applications with successive design steps are for instance structural optimization, reliability analysis or structural damage analysis. In many cases hundreds or even thousands of different design configurations are investigated and the state equation has to be solved for each design step. The repeated structural analysis of large problems involve significant computational effort.

An efficient reanalysis method can be used in order to reduce the overall computational cost. The goal of classical reanalysis procedures is to evaluate the change in the state variables for some given design modifications efficiently and with sufficiently accuracy, without directly solving the set of modified equations of the changed problem. Reanalysis techniques for the computation of changes in the state variables due to given design changes have been extensively studied in the literature, see e.g. $[2,8,6,10,16,7,13]$, among many others. It has been formulated for linear statics and dynamic problems. Furthermore, reanalysis methods have been applied to sensitivity analysis and optimization problems, see e.g. [9, 15, 1].

Very often we are interested only in certain quantities of interest $J$ and so-called goal-oriented or duality techniques or adjoint state methods can be used to compute $J$. In structural mechanics this is known as the concept of influence functions, see e.g. [5]. The quantities of interest could be point values, e.g. a displacement component or a stress component at a point, or some integral value.

Within this contribution a method for the prediction of the change in a quantity of interest $\Delta J$ due to structural modifications by using the changes in the primal and dual solutions is presented. The changes in the primal and dual solutions are computed by using the residual increment approximation (RIA) method [13]. This reanalysis procedure is based on a formulation in terms of residual increments. In 
contrast to other existing reanalysis methods (e.g. the CA method [10]), which are based on the evaluation of changed stiffness matrices, only residual vectors have to be computed and stored. Hence, this yields an efficient goal-oriented reanalysis method in order to compute the change in the quantities of interest due to given design modifications with sufficiently accuracy, without directly solving the set of modified equations of the changed problem.

\section{Formulation of goal-oriented analysis}

\subsection{The quantities of interest}

In goal-oriented or duality techniques we are interested in an objective functional or quantity of interest $J$, which can be every functional value which corresponds in some sense to the solution $\mathbf{u}$. We consider a quantity of interest $J(\mathbf{s})(\mathbf{u})$ which depends on the state variables $\mathbf{u}$ and on some design variables $\mathbf{s}$, i.e. parameter which are describing the cross-sections, the geometry, the topology or material parameter etc. The quantities of interest could be point values, e.g. a displacement component $u_{i}(\mathbf{X})$ or a stress component $\sigma_{i j}(\mathbf{X})$ at a point $\mathbf{X}$. Furthermore, it can be some integral value, e.g.

$$
J(\mathbf{s})(\mathbf{u})=u_{i}(\mathbf{X}) \quad \text { or } \quad J(\mathbf{s})(\mathbf{u})=\sigma_{i j}(\mathbf{X}) \quad \text { or } \quad J(\mathbf{s})(\mathbf{u})=\int_{A} \sigma_{i j} \mathrm{~d} A .
$$

\subsection{The primal and dual (adjoint) problems}

The state $\mathbf{u}$ is determined by the primal problem in terms of a residual $\mathbf{R}(\mathbf{s})(\mathbf{u})$. In the linear case the

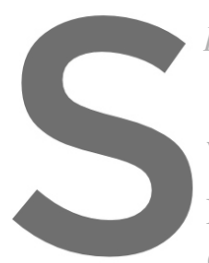
primal solution u is given from

where $\mathbb{K}$ is the stiffness $m$

For a chosen $J(\mathbf{s})(\mathbf{u})$, the corresponding dual or Green's function $\mathbf{z}$ is determined by the so-called
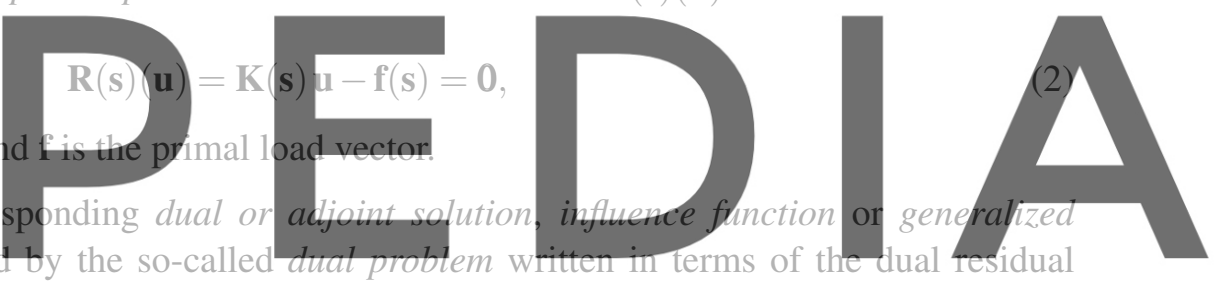

vector $\mathbb{R}^{*}(\mathbf{s})(\mathbf{z})$. The dual solution $\mathbf{z}$ is given from

Register for free at https//www.scipedia.com to download the version without the watermark

$$
\mathbf{R}^{*}(\mathbf{s})(\mathbf{z})=\mathbf{K}^{T}(\mathbf{s}) \mathbf{z}-\mathbf{j}(\mathbf{s})=\mathbf{0},
$$

where

$$
\mathbf{j}:=\left(\frac{\partial J}{\partial \mathbf{u}}\right)^{T}
$$

is the so-called dual load vector. For self-adjoint problems we have $\mathbf{K}=\mathbf{K}^{T}$. In the general nonlinear case, the dual problem is formulated at a given deformed state, i.e. the dual residual depends also on $\mathbf{u}$, see e.g. [12].

The residual vectors $\mathbf{R}$ and $\mathbf{R}^{*}$ as well as the quantities of interest $J$ are linear in $\mathbf{u}$ and $\mathbf{z}$, respectively, but in the general case nonlinear in $\mathbf{s}$.

\subsection{Computing the quantity of interest}

The quantity of interest $J$ can be computed in two different ways. In the classical way the primal problem $\mathbf{R}(\mathbf{s})(\mathbf{u})=\mathbf{0}$ is solved and $J(\mathbf{s})(\mathbf{u})$ can be computed in a post-processing step. 
Alternatively, the dual solution $\mathbf{z}$ can be used, which can have a big advantage. With the primal and dual problems $\mathbf{f}=\mathbf{K u}$ and $\mathbf{j}=\mathbf{K}^{T} \mathbf{z}$ we have

$$
\begin{aligned}
J(\mathbf{s})(\mathbf{u})=\mathbf{j}^{T} \mathbf{u}=\mathbf{u}^{T} \mathbf{j} & =\mathbf{u}^{T} \mathbf{K}^{T} \mathbf{z} \\
& =\mathbf{z}^{T} \mathbf{K} \mathbf{u}=\mathbf{z}^{T} \mathbf{f}=\mathbf{f}^{T} \mathbf{z} .
\end{aligned}
$$

Finally, $J$ is simply given as

$$
J(\mathbf{s})(\mathbf{u})=\mathbf{j}^{T} \mathbf{u}
$$

or alternatively from

$$
J(\mathbf{s})(\mathbf{u})=\mathbf{f}^{T} \mathbf{z} .
$$

The big advantage of the formulation (7) can be summarized as follows: If the dual solution $\mathbf{z}$ is known, the quantity of interest $J(\mathbf{s})(\mathbf{u})$ can be computed for arbitrary primal load vectors $\mathbf{f}$ just by a simple scalar product of $\mathbf{z}$ and $\mathbf{f}$. This is for instance used in structural mechanics in the context of influence functions in order to compute $J$ for many different load cases.

\section{Goal-oriented reanalysis}

Reanalysis is used in many fields which are concerned with design modifications. Let $\mathbf{s}_{0}$ be a given initial design and let $\mathbf{u}_{0}$ the corresponding solution of (2). Assume a changed design $\mathbf{s}_{c}=\mathbf{s}_{0}+\Delta \mathbf{s}$ and let $\mathbf{u}_{c}$ be the corresponding solution. Furthermore, let $J\left(\mathbf{s}_{0}\right)\left(\mathbf{u}_{0}\right)$ be the value of $J$ for the initial design $\mathbf{s}_{0}$ and let $J\left(\mathbf{s}_{c}\right)\left(\mathbf{u}_{c}\right)$ be the value of $J$ for a given changed design $\mathbf{S}_{c}$

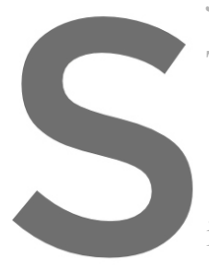

The general goal-oriented reanalysis problem can

in the quantity of interest due to given design chat ge
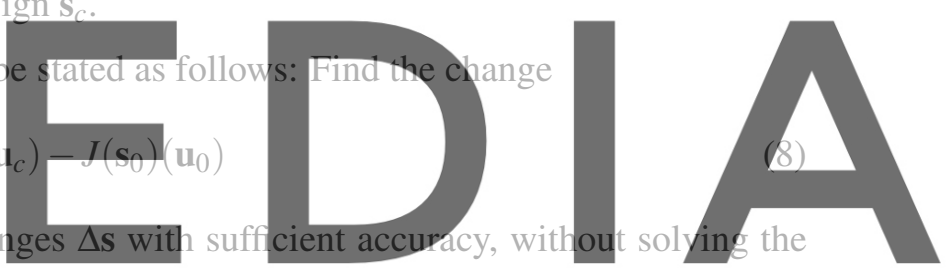

complete modified equations.

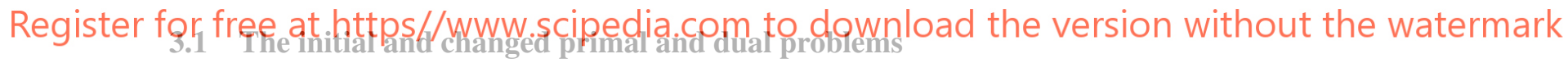

The primal and dual problems for the initial and changed designs according to (2) and (3) are given as

$$
\begin{aligned}
& \mathbf{R}\left(\mathbf{s}_{0}\right)\left(\mathbf{u}_{0}\right)=\mathbf{K}_{0} \mathbf{u}_{0}-\mathbf{f}_{0}=\mathbf{0} \\
& \mathbf{R}^{*}\left(\mathbf{s}_{0}\right)\left(\mathbf{z}_{0}\right)=\mathbf{K}_{0}^{T} \mathbf{z}_{0}-\mathbf{j}_{0}=\mathbf{0} .
\end{aligned}
$$

and

$$
\begin{aligned}
& \mathbf{R}\left(\mathbf{s}_{c}\right)\left(\mathbf{u}_{c}\right)=\mathbf{K}_{c} \mathbf{u}_{c}-\mathbf{f}_{c}=\mathbf{0} \\
& \mathbf{R}^{*}\left(\mathbf{s}_{c}\right)\left(\mathbf{z}_{c}\right)=\mathbf{K}_{c}^{T} \mathbf{z}_{c}-\mathbf{j}_{c}=\mathbf{0},
\end{aligned}
$$

where $\mathbf{K}_{0}=\mathbf{K}\left(\mathbf{s}_{0}\right), \mathbf{K}_{c}=\mathbf{K}\left(\mathbf{s}_{c}\right), \mathbf{f}_{0}=\mathbf{f}\left(\mathbf{s}_{0}\right), \mathbf{f}_{c}=\mathbf{f}\left(\mathbf{s}_{c}\right), \mathbf{j}_{0}=\mathbf{j}\left(\mathbf{s}_{0}\right)$ and $\mathbf{j}_{c}=\mathbf{j}\left(\mathbf{s}_{c}\right)$. 


\subsection{The change in the quantity of interest}

The change in the quantity of interest $\Delta J$ can be computed in two different ways. In the first way we use a formulation with $\mathbf{u}_{0}$ and the change $\Delta \mathbf{u}=\mathbf{u}_{c}-\mathbf{u}_{0}$. With (6) we obtain after some manipulations

$$
\begin{aligned}
\Delta J & =J\left(\mathbf{s}_{c}\right)\left(\mathbf{u}_{c}\right)-J\left(\mathbf{s}_{0}\right)\left(\mathbf{u}_{0}\right)=\mathbf{j}_{c}^{T} \mathbf{u}_{c}-\mathbf{j}_{0}^{T} \mathbf{u}_{0} \\
& =\Delta \mathbf{j}^{T} \mathbf{u}_{c}+\mathbf{j}_{0}^{T} \Delta \mathbf{u} \\
& =\Delta \mathbf{j}^{T} \mathbf{u}_{0}+\mathbf{j}_{c}^{T} \Delta \mathbf{u} .
\end{aligned}
$$

The change $\Delta J$ depends on $\Delta \mathbf{j}=\mathbf{j}_{c}-\mathbf{j}_{0}$ and the change $\Delta \mathbf{u}$.

Alternatively, the change in $J$ can be expressed in terms of $\mathbf{z}_{0}$ and the change $\Delta \mathbf{z}=\mathbf{z}_{c}-\mathbf{z}_{0}$. By using (7) we obtain

$$
\begin{aligned}
\Delta J & =J\left(\mathbf{s}_{c}\right)\left(\mathbf{u}_{c}\right)-J\left(\mathbf{s}_{0}\right)\left(\mathbf{u}_{0}\right)=\mathbf{f}_{c}^{T} \mathbf{z}_{c}-\mathbf{f}_{0}^{T} \mathbf{z}_{0} \\
& =\Delta \mathbb{f}^{T} \mathbb{Z}_{c}+\mathbf{f}_{0}^{T} \Delta \mathbf{z} \\
& =\Delta \mathbf{f}^{T} \mathbb{Z}_{0}+\mathbb{f}_{c}^{T} \Delta \mathbf{z} .
\end{aligned}
$$

In this formulation, the change $\Delta J$ depends on $\Delta \mathbf{f}=\mathbf{f}_{c}-\mathbf{f}_{0}$ and the change $\Delta \mathbf{z}$.

Finally, according to (6) and (7) we obtain the two equivalent relations (13) and (14) in order to express the change $\Delta J$.

\section{The big advantage of the \\ and the change $\Delta \mathrm{z}$ for a \\ for arbitrary primal load \\ load cases just by evaluat \\ 4 Computing the change in the equantity of int
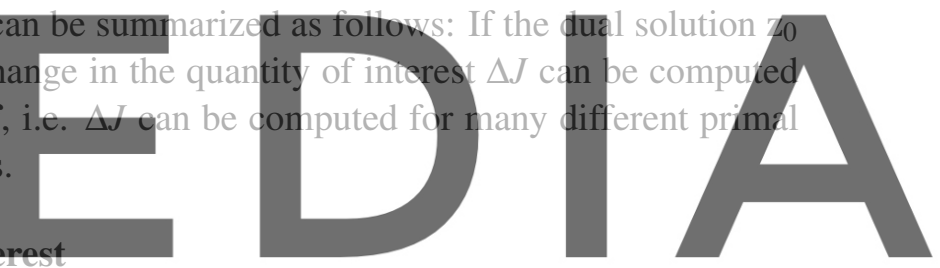 \\ Register In order to compute $J$ we have, to eyaluate (13) or (14). The changes in the primal and dual loads $\Delta f$ or free at https cwww. scipedia.com to dow \\ approximations of the increments $\Delta \mathbf{u}$ or $\Delta \mathbf{z}$ with sufficient accuracy. This can be done by using reanalysis methods. A general efficient reanalysis method for the computation of an approximation of $\Delta \mathbf{u}$ based on residual increment approximations (RIA method) has been presented in [13]. This method is in the following used in order to compute the changes $\Delta \mathbf{u}$ and $\Delta \mathbf{z}$ with sufficient accuracy, without solving the complete modified equations.}

\subsection{Reanalysis for the primal problem}

The starting point for the reanalysis method is the residual of the changed problem $\mathbf{R}\left(\mathbf{s}_{c}\right)\left(\mathbf{u}_{c}\right)$ defined in (11). Although, the problem is linear in $\mathbf{u}$, the residual is in the general case nonlinear in $\mathbf{s}$, i.e.

$$
\mathbf{R}\left(\mathbf{s}_{c}\right)\left(\mathbf{u}_{c}\right) \neq \mathbf{R}\left(\mathbf{s}_{0}\right)\left(\mathbf{u}_{c}\right)+\mathbf{R}(\Delta \mathbf{s})\left(\mathbf{u}_{c}\right) .
$$

The changed problem (11) can be expressed in terms of the initial design $\mathbf{s}_{0}$ as

$$
\mathbf{R}\left(\mathbf{s}_{c}\right)\left(\mathbf{u}_{c}\right)=\mathbf{R}\left(\mathbf{s}_{0}\right)\left(\mathbf{u}_{c}\right)+\Delta_{s} \mathbf{R}(\Delta \mathbf{s})\left(\mathbf{u}_{c}\right)=\mathbf{0} .
$$


Hence, the residual increment with respect to $\mathbf{s}$ is given as

$$
\Delta_{s} \mathbf{R}(\Delta \mathbf{s})\left(\mathbf{u}_{c}\right)=\mathbf{R}\left(\mathbf{s}_{c}\right)\left(\mathbf{u}_{c}\right)-\mathbf{R}\left(\mathbf{s}_{0}\right)\left(\mathbf{u}_{c}\right) .
$$

Furthermore, we obtain with $\mathbf{R}\left(\mathbf{s}_{0}\right)\left(\mathbf{u}_{0}\right)=\mathbf{0}$ for the first term on the right side in (16) the relation

$$
\begin{aligned}
\mathbf{R}\left(\mathbf{s}_{0}\right)\left(\mathbf{u}_{c}\right) & =\mathbf{R}\left(\mathbf{s}_{0}\right)\left(\mathbf{u}_{0}\right)+\mathbf{R}\left(\mathbf{s}_{0}\right)(\Delta \mathbf{u})=\mathbf{K}_{0} \mathbf{u}_{0}-\mathbf{f}_{0}+\mathbf{K}_{0} \Delta \mathbf{u} \\
& =\mathbf{K}_{0} \Delta \mathbf{u} .
\end{aligned}
$$

Finally, Eq. (16) leads to

$$
\begin{aligned}
\mathbf{K}_{0} \Delta \mathbf{u} & =-\Delta_{s} \mathbf{R}(\Delta \mathbf{s})\left(\mathbf{u}_{c}\right) \\
& =-\left[\mathbf{R}\left(\mathbf{s}_{c}\right)\left(\mathbf{u}_{c}\right)-\mathbf{R}\left(\mathbf{s}_{0}\right)\left(\mathbf{u}_{c}\right)\right] \\
& =-\left[\mathbf{R}\left(\mathbf{s}_{c}\right)\left(\mathbf{u}_{c}\right)-\mathbf{K}_{0} \Delta \mathbf{u}\right] .
\end{aligned}
$$

This can be expressed as the recurrence relation

$$
\mathbf{K}_{0} \Delta \mathbf{u}_{i}=-\mathbf{Q}\left(\Delta \mathbf{u}_{i-1}\right),
$$

\section{where}

$$
\begin{aligned}
\mathbf{Q}\left(\Delta \mathbf{u}_{i-1}\right) & =\mathbb{R}\left(\mathbf{s}_{C}\right)\left(\mathbf{u}_{0}+\Delta \mathbf{u}_{i-1}\right)-\mathbb{K}_{0} \Delta \mathbf{u}_{i-1} \\
& =\mathbb{R}\left(\mathbf{s}_{c}\right)\left(\mathbf{u}_{0}+\Delta \mathbf{u}_{i-1}\right)+\mathbf{Q}\left(\Delta \mathbf{u}_{i-2}\right) .
\end{aligned}
$$
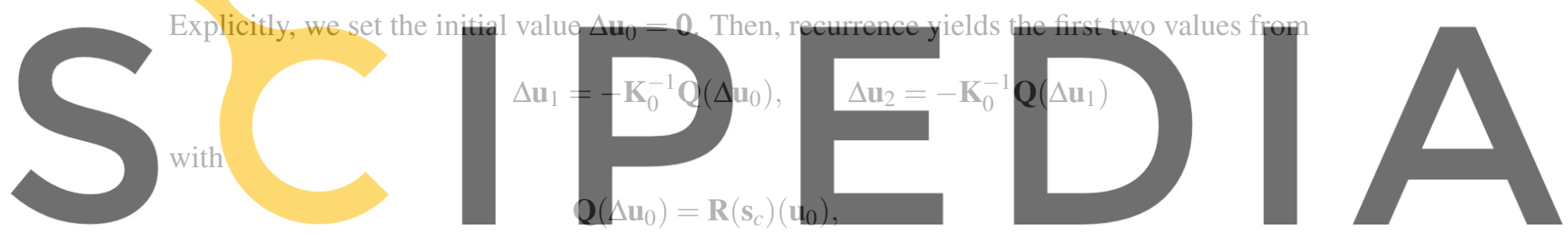

Register for free at https//wwww.scipedia.com to download the version without the watermark

$$
\Delta \mathbf{u}_{i}=-\mathbb{K}_{0}^{-1} \mathbf{Q}\left(\Delta \mathbf{u}_{i-1}\right) \quad i=3,4, \ldots n .
$$

After $n$ iterations, the approximation of the state $\tilde{\mathbf{u}}_{c}$ for the changed design $\mathbf{s}_{c}$ is obtained as

$$
\tilde{\mathbf{u}}_{c}=\mathbf{u}_{0}+\Delta \mathbf{u}_{n} .
$$

The stiffness matrix $\mathbf{K}_{0}$ is the same as used for the solution of the initial design and therefore usually already given in decomposed form. Therefore, the computation of $\Delta \mathbf{u}_{i}$ in (20) requires just forward and backward substitution. Within the reanalysis method only residual vectors have to be computed and stored.

The reanalysis procedure adapted from Eq. 20 is a local approximation based on information calculated at a single point $\left(\mathbf{s}_{0}, \mathbf{u}_{0}\right)$. The results can be further improved by using a vector-valued rational approximation method introduced in [14] and applied to linear reanalysis problems in [16].

This method is used within the numerical examples in the present paper. Details about this method as well as the overall algorithm of the reanalysis method are given in [13]. 


\subsection{Reanalysis for the dual problem}

In the same way as for the primal problem, we can formulate a reanalysis method for the dual problem in order to evaluate (14).

The changed problem (12) can be expressed in terms of the initial design $\mathbf{s}_{0}$ as

$$
\mathbf{R}^{*}\left(\mathbf{s}_{c}\right)\left(\mathbf{z}_{c}\right)=\mathbf{R}^{*}\left(\mathbf{s}_{0}\right)\left(\mathbf{z}_{c}\right)+\Delta_{s} \mathbf{R}^{*}(\Delta \mathbf{s})\left(\mathbf{z}_{c}\right)=\mathbf{0} .
$$

Furthermore, we obtain with $\mathbf{R}^{*}\left(\mathbf{s}_{0}\right)\left(\mathbf{z}_{0}\right)=\mathbf{0}$ for the first term on the right side in (24) the relation

$$
\begin{aligned}
\mathbf{R}^{*}\left(\mathbf{s}_{0}\right)\left(\mathbf{z}_{c}\right) & =\mathbf{R}^{*}\left(\mathbf{s}_{0}\right)\left(\mathbf{z}_{0}\right)+\mathbf{R}^{*}\left(\mathbf{s}_{0}\right)(\Delta \mathbf{z})=\mathbf{K}_{0}^{T} \mathbf{z}_{0}-\mathbf{j}_{0}+\mathbf{K}_{0}^{T} \Delta \mathbf{z} \\
& =\mathbf{K}_{0}^{T} \Delta \mathbf{z} .
\end{aligned}
$$

Finally, Eg. (24) leads to

$$
\begin{aligned}
\mathbf{K}_{0}^{T} \Delta \mathbf{z} & =-\Delta_{s} \mathbf{R}^{*}(\Delta \mathbf{s})\left(\mathbf{z}_{c}\right) \\
& =-\left[\mathbf{R}^{*}\left(\mathbf{s}_{c}\right)\left(\mathbf{z}_{c}\right)-\mathbf{R}^{*}\left(\mathbf{s}_{0}\right)\left(\mathbf{z}_{c}\right)\right] \\
& =-\left[\mathbf{R}^{*}\left(\mathbf{s}_{c}\right)\left(\mathbf{z}_{c}\right)-\mathbf{K}_{0}^{T} \Delta \mathbf{z}\right]
\end{aligned}
$$

This can be expressed as the recurrence relation in form of
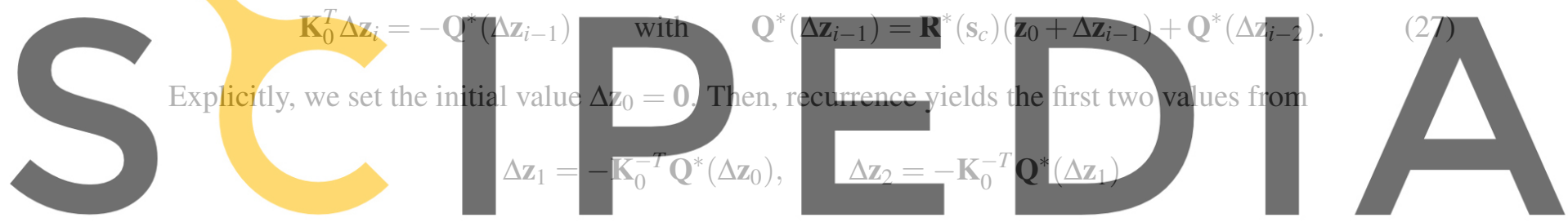

with

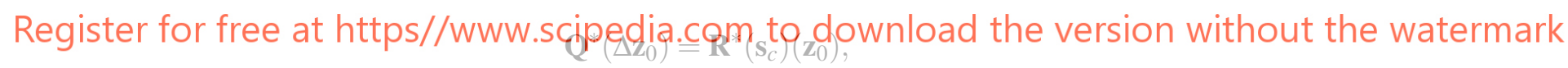

$$
\mathbf{Q}^{*}\left(\Delta \mathbf{z}_{1}\right)=\mathbf{R}^{*}\left(\mathbf{s}_{c}\right)\left(\mathbf{z}_{0}+\Delta \mathbf{z}_{1}\right)+\mathbf{Q}^{*}\left(\Delta \mathbf{z}_{0}\right) .
$$

For all other values we have

$$
\Delta \mathbf{z}_{i}=-\mathbf{K}_{0}^{-T} \mathbf{Q}^{*}\left(\Delta \mathbf{z}_{i-1}\right) \quad i=3,4, \ldots n .
$$

After $n$ iterations, the approximation of the dual solution $\tilde{\mathbf{z}}_{c}$ for the changed design $\mathbf{s}_{c}$ is obtained as

$$
\tilde{\mathbf{z}}_{c}=\mathbf{z}_{0}+\Delta \mathbf{z}_{n} .
$$

In the same way as for the primal problem, the results can be further improved by using a vector-valued rational approximation method. This is used within the numerical examples. 


\subsection{First-order adjoint sensitivity relation}

In many applications, the classical first-order approximation (FOA) is used in order to predict the changes in the state variables or a quantities of interest due to design modifications, see e.g. [11, 12]. The results are valid only for very small design changes. For completeness, within this study we compare the proposed reanalysis method with the classical FOA.

In order to compute the changes in a quantity of interest so-called adjoint sensitivity analysis can be used, see e.g. [4]. For a given fixed design change $\Delta \mathbf{s}$ we obtain the first-order approximation $\Delta \tilde{J}$ for the change in the quantity of interest in form of

$$
\Delta \tilde{J}=\left[\frac{\partial J\left(\mathbf{s}_{0}\right)\left(\mathbf{u}_{0}\right)}{\partial \mathbf{s}}-\mathbf{z}_{0}^{T} \mathbf{P}_{0}\right] \Delta \mathbf{s} .
$$

The matrix $\mathbf{P}_{0}$ is the co-called pseudo load matrix and defined as

$$
\mathbb{P}_{0}=\mathbb{P}\left(\mathrm{s}_{0}\right)\left(\mathbf{u}_{0}\right)=\frac{\partial \mathbb{R}}{\partial \mathrm{s}}\left(\mathrm{s}_{0}\right)\left(\mathbf{u}_{0}\right)
$$

see e.g. $[11,12,3]$ for details and explicit formulations. The advantage of the first-order approximation (30) is that the relation depends only on the known initial primal and dual solutions $\mathbf{u}_{0}$ and $\mathbf{z}_{0}$, i.e. the changes in the primal and dual solutions are not required. The disadvantage of this method is that it requires the derivatives with respect to the design variables, i.e. we have to compute $\frac{\partial J}{\partial s}$ and $\mathbb{P}=\frac{\partial \mathbf{R}}{\partial s}$.

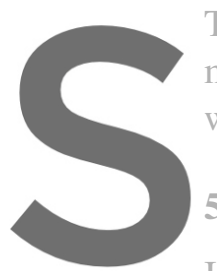
This can be very difficul method and used in many within the numerical examples.

5 Numerical examples

In the above sections we have considered three net
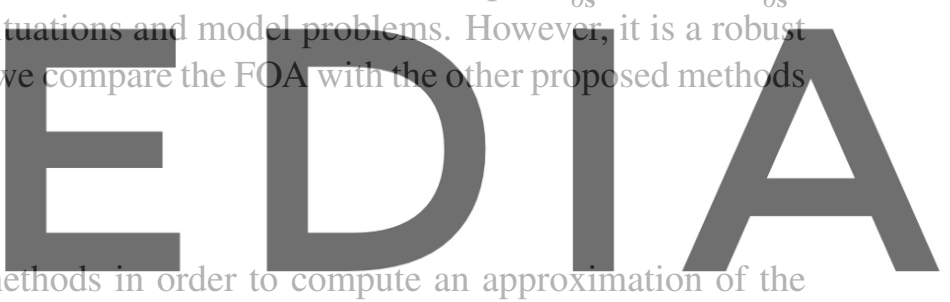

change $\Delta J=J\left(\mathbf{s}_{c}\right)\left(\mathbf{u}_{c}\right)-J\left(\mathbf{s}_{0}\right)\left(\mathbf{u}_{0}\right)$ for a given fixed design change $\Delta \mathbf{s}$. The methods are summarized in

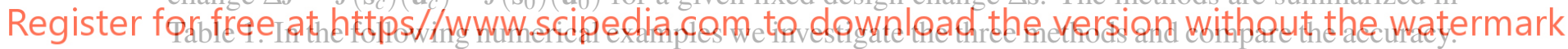

For all numerical examples we consider the model problem of linear elasticity.

Table 1: Summary of different methods in order to compute approximations of the change $\Delta J$

\begin{tabular}{lll}
\hline Method & Discrete formulation & \\
\hline 1 & $\Delta J=\Delta \mathbf{j}^{T} \mathbf{u}_{0}+\mathbf{j}_{c}^{T} \Delta \mathbf{u}$ & (see Eq. 13) \\
\hline 2 & $\Delta J=\Delta \mathbf{f}^{T} \mathbf{z}_{0}+\mathbf{f}_{c}^{T} \Delta \mathbf{z}$ & (see Eq. 14) \\
\hline 3 & $\Delta \tilde{J}=\left[\frac{\partial J}{\partial \mathbf{s}}-\mathbf{z}_{0}^{T} \mathbf{P}_{0}\right] \Delta \mathbf{s}$ & (see Eq. 30) \\
\hline
\end{tabular}

Approximations of the changes in the primal and dual solutions $\Delta \mathbf{u}$ and $\Delta \mathbf{z}$ required in methods 1 and 2 are computed by using the reanalysis method from (20) and (27), respectively. Furthermore, the accuracies of $\Delta \mathbf{u}$ and $\Delta \mathbf{z}$ are improved by using a vector-valued rational approximation method as described in [13]. 


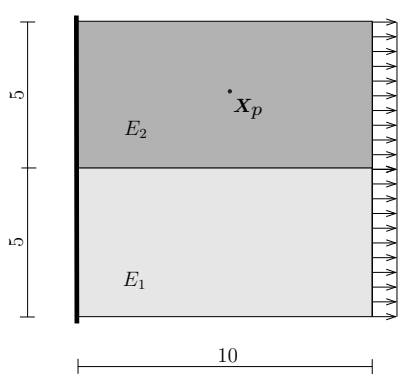

(a) primal problem

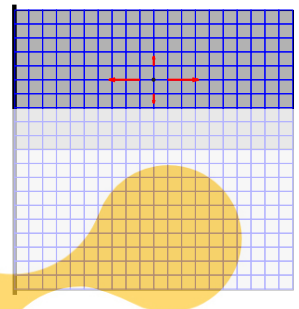

(d) dual problem

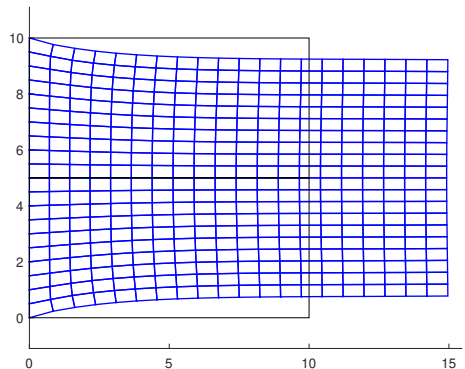

(b) initial primal solution $\mathbf{u}_{0}$

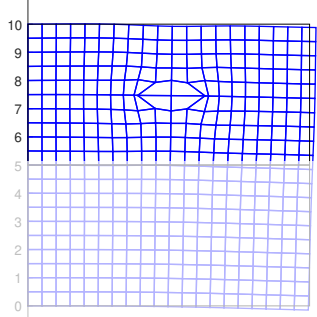

(e) initial dual solution $\mathbf{z}_{0}$

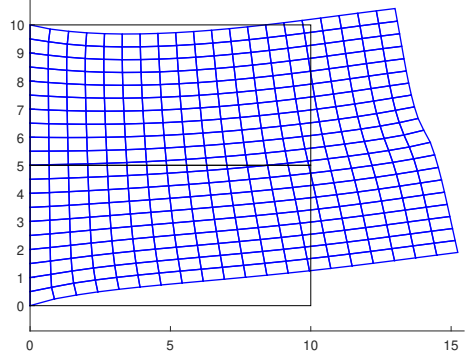

(c) changed solution $\mathbf{u}_{c}\left(\Delta E_{2}=50\right)$

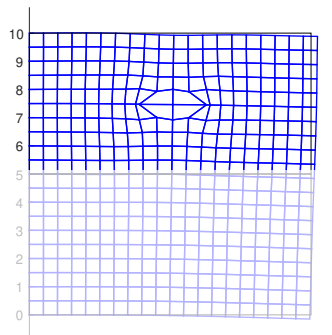

(f) changed solution $\mathbf{z}_{c}\left(\Delta E_{2}=50\right)$

Figure 1: Bi-material solid under tension: The design variables are the Young's moduli $E_{1}$ and $E_{2}$ in the two domains, i.e. $\mathrm{s}=\left[E_{1} E_{2}\right]^{T}$ with the initial design $\mathrm{s}_{0}=\left[\begin{array}{lll}100 & 100\end{array}\right]^{T}$. A large design change $\Delta \mathrm{s}=\left[\begin{array}{ll}0 & 50\end{array}\right]^{T}$ is investigated.

The quantity of interest is the stress component $\sigma_{x x}$ at point $\mathbf{X}_{p}$, i.e $J(\mathrm{~s})(\mathbf{u})=\sigma_{m}\left(\mathbf{X}_{p}\right)$ The dual load case (red
arrows in (d)) causes an approximation of a unjt dislocation at point $\mathbf{X}_{p}$.
F.1 Bi-material solid under tension clamped on the left side and loaded by traction $t_{x}=50$. The design variables are the Young's moduli $E_{1}$ Register fardfee atenttps fhwww.scipedia.com to download the version without the watermark

$$
\mathrm{S}=\left[\begin{array}{ll}
E_{1} & E_{2}
\end{array}\right]^{T} \quad \text { and } \quad \Delta \mathrm{s}=\left[\Delta E_{1} \Delta E_{2}\right]^{T} .
$$

For simplicity, we consider only changes in the second variable $E_{2}$ and we keep $E_{1}$ constant. For the initial design $\mathbf{s}_{0}$ we chose $E_{1}=E_{2}=100$. The Poisson's ratio for both domains is $v=0.3$ and the finite element discretization consists of classical bilinear Q4 elements.

The quantity of interest is the stress component $\sigma_{x x}$ at point $\mathbf{X}_{p}$, i.e. $J(\mathbf{s})(\mathbf{u})=\sigma_{x x}\left(\mathbf{X}_{p}\right)$. The dual load case $\mathbf{j}$ (red arrows in Fig. 1d causes an approximation of a unit dislocation at point $\mathbf{X}_{p}$. The primal and dual solutions for the initial design are given in Fig. 1b and Fig. 1e. In this example a large change in the Young's moduli is considered, i.e. we investigate a design change $\Delta \mathbf{s}=\left[\begin{array}{ll}0 & 50\end{array}\right]^{T}$. This yields a large change in the primal solution, see Fig. 1c. The change in the dual solution due to the design change is relatively small, see Fig. 1f.

The values of $J$ for the initial and changed designs as well as the exact change are given as

$$
J\left(\mathbf{s}_{0}\right)\left(\mathbf{u}_{0}\right)=50.3917, \quad J\left(\mathbf{s}_{c}\right)\left(\mathbf{u}_{c}\right)=52.6092, \quad \Delta J=\Delta \sigma_{x x}\left(\mathbf{X}_{p}\right)=2.2175 .
$$


Table 2: Bi-material solid under tension $\left(J(\mathbf{s})(\mathbf{u})=\sigma_{x x}\left(\mathbf{X}_{p}\right)\right)$ : Accuracy of the different methods (see Table 1). The results are given for different $n$, which is the number of iterations used within the reanalysis in methods 1 and 2. The relative errors are given w.r.t. the exact change $\Delta J=\Delta \sigma_{x x}\left(\mathbf{X}_{p}\right)=2.2175$.

Results for $n=1$ :

\begin{tabular}{lll}
\hline Method & Approximation of $\Delta J$ & rel. error $[\%]$ \\
\hline 1 & 2.1349 & 3.7241 \\
\hline 2 & 2.1619 & 2.5076 \\
\hline 3 & 2.7415 & 23.628 \\
\hline
\end{tabular}

$\underline{\text { Results for } n=3 \text { : }}$

\begin{tabular}{lll}
\hline Method & Approximation of $\Delta J$ & rel. error $[\%]$ \\
\hline 1 & 2.2179 & 0.0164 \\
\hline 2 & 2.2176 & 0.0027 \\
3 & 2.7415 & 23.628
\end{tabular}

Results for $n=2$ :

\begin{tabular}{|c|c|c|}
\hline Method & Approximation of $\Delta J$ & rel. error $[\%]$ \\
\hline 1 & 2.1878 & 1.3397 \\
\hline 2 & 2.2175 & 0.0018 \\
\hline 3 & 2.7415 & 23.628 \\
\hline
\end{tabular}

$\underline{\text { Results for } n=4 \text { : }}$

\begin{tabular}{lll}
\hline Method & Approximation of $\Delta J$ & rel. error $[\%]$ \\
\hline 1 & 2.2175 & 0.0014 \\
\hline 2 & 2.2175 & 0.0001 \\
\hline 3 & 2.7415 & 23.628 \\
\hline
\end{tabular}

Approximations of the changes in the quantity of interest are computed by using the three methods given in Table 1. The results are stated in Table 2.

Method 1 and 2 yield $y$ relation and yields for method 2 outperforms

$\Delta \mathrm{s}$ is relatively small an the reanalysis method.

Therefore, we need morc
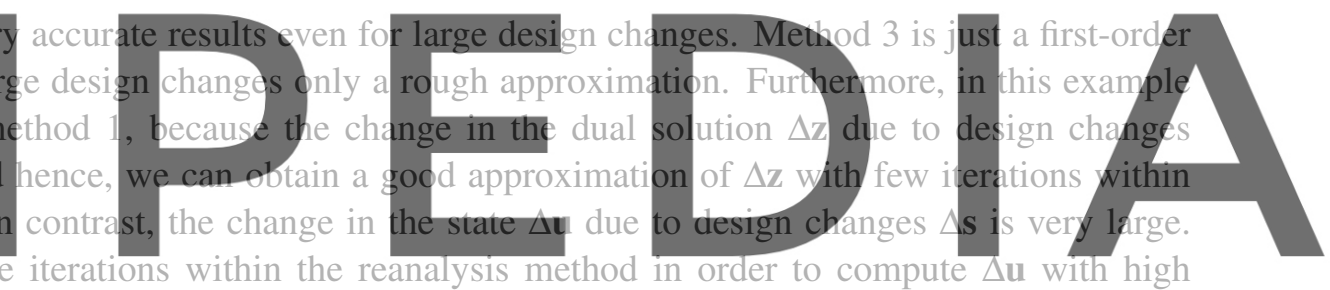
accuracy.

\subsection{L-shaped domain with geometry changes}

Within this example a L-shaped domain with shape changes is investigated. The body is clamped on the left and top side and loaded by traction $t_{y}=-0.5$, see Fig. 2a. The Poisson's ratio and the Young's modulus are chosen as $v=0.3$ and $E=10$. The problem is modeled by using standard Q4 elements.

The design variable is the height $H_{1}$ of the left boundary. This results in a linear change of the lower boundary as indicated in Fig. 2b. The nodes of the fe mesh are moved due to the changes of the lower boundary by using a linear design velocity field and almost all fe nodes are effected by design modifications. The initial and changed solution for a change $\Delta H_{1}=2$ are shown in Fig. $3 \mathrm{~b}$ and Fig. 3c, respectively.

The quantity of interest is the displacement component $u_{x}$ at point $\mathbf{X}_{p}$, i.e. $J(\mathbf{s})(\mathbf{u})=u_{x}\left(\mathbf{X}_{p}\right)$. The dual load case (red arrow in Fig. 3d) is a unit load at point $\mathbf{X}_{p}$ in $x$-direction.

The values of $J$ for the initial and changed designs as well as the exact change are given as

$$
J\left(\mathbf{s}_{0}\right)\left(\mathbf{u}_{0}\right)=-1.667, \quad J\left(\mathbf{s}_{c}\right)\left(\mathbf{u}_{c}\right)=-1.1118, \quad \Delta J=\Delta u_{x}\left(\mathbf{X}_{p}\right)=0.55514 .
$$




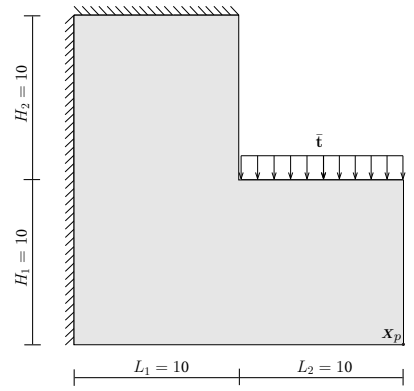

(a) initial design $\mathbf{s}_{0}$

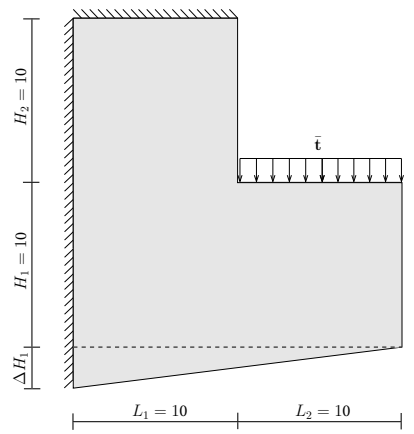

(b) changed design $\mathbf{s}_{c}=\mathbf{s}_{0}+\Delta \mathbf{s}$

Figure 2: L-shaped domain with geometry changes: The design variable is the height $H_{1}$ of the left boundary.

Approximations of the changes in the quantity of interest are computed by using the tree methods given in Table 1. The results are stated in Table 3. Method 1 and 2 yield again very accurate results even for large design changes. Method 3 yields for large design changes only a rough approximation.
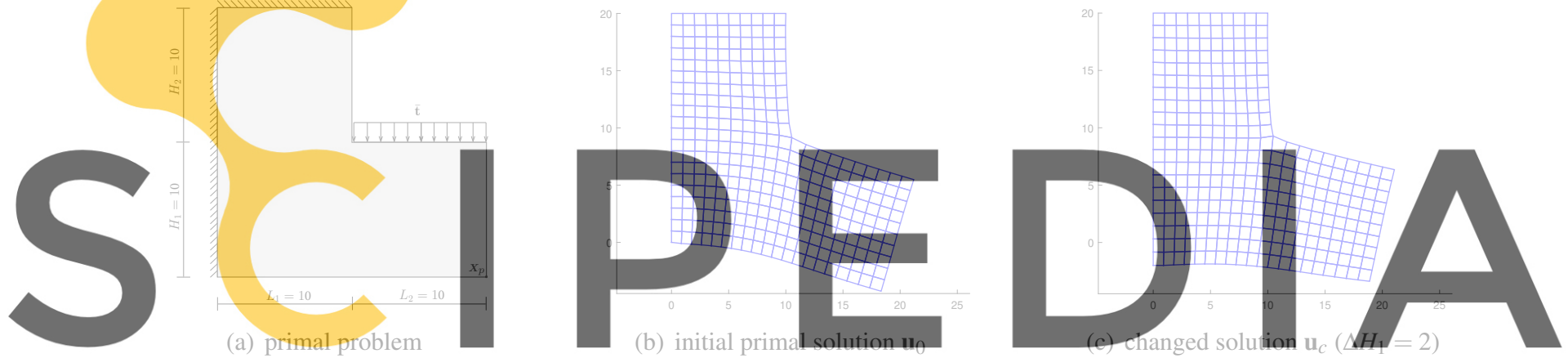

\section{Register for free at https//www.scipedia.com to download the version without the watermark}

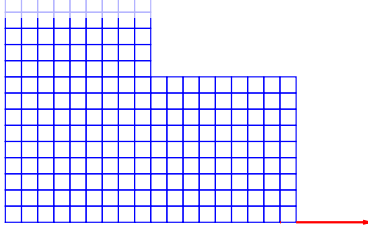

(d) dual problem

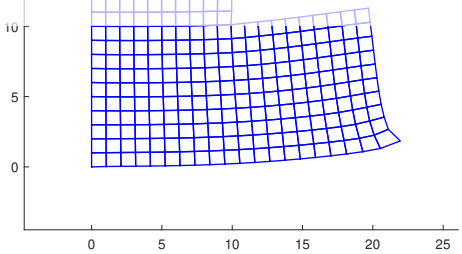

(e) initial dual solution $\mathbf{z}_{0}$

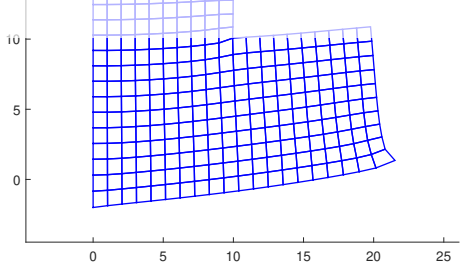

(f) changed solution $\mathbf{z}_{c}\left(\Delta H_{1}=2\right)$

Figure 3: L-shaped domain with geometry changes: The design variable is the height $H_{1}$ of the left boundary. A large design change $\Delta H_{1}=2$ is investigated. The quantity of interest is the displacement component $u_{x}$ at point $\mathbf{X}_{p}$, i.e. $J(\mathbf{s})(\mathbf{u})=u_{x}\left(\mathbf{X}_{p}\right)$. The dual load case (red arrow in (d)) is a unit load at point $\mathbf{X}_{p}$ in $x$-direction. 
Table 3: L-shaped domain with geometry changes $\left(J(\mathbf{s})(\mathbf{u})=u_{x}\left(\mathbf{X}_{p}\right)\right.$ ): Accuracy of the different methods (see Table 1). The results are given for different $n$, which is the number of iterations used within the reanalysis in methods 1 and 2. The relative errors are given w.r.t. the exact change $\Delta J=\Delta u_{x}\left(\mathbf{X}_{p}\right)=0.55514$.

Results for $n=1$ :

\begin{tabular}{lll}
\hline Method & Approximation of $\Delta J$ & rel. error [\%] \\
\hline 1 & 0.5531 & 0.3680 \\
\hline 2 & 0.5581 & 0.5275 \\
\hline 3 & 0.6899 & 24.267 \\
\hline
\end{tabular}

$\underline{\text { Results for } n=3 \text { : }}$

\begin{tabular}{lll}
\hline Method & Approximation of $\Delta J$ & rel. error $[\%]$ \\
\hline 1 & 0.5552 & 0.0152 \\
\hline 2 & 0.5551 & 0.0010 \\
\hline 3 & 0.6897 & 24.267 \\
\hline
\end{tabular}

\begin{tabular}{lll}
\multicolumn{3}{l}{ Results for $n=2$ : } \\
\hline Method & Approximation of $\Delta J$ & rel. error [\%] \\
\hline 1 & 0.5568 & 0.3018 \\
\hline 2 & 0.5561 & 0.1797 \\
\hline 3 & 0.6899 & 24.267 \\
\hline
\end{tabular}

\begin{tabular}{lll}
\multicolumn{2}{l}{ Results for $n=4:$} \\
\hline Method & Approximation of $\Delta J$ & rel. error $[\%]$ \\
\hline 1 & 0.5551 & 0.0007 \\
\hline 3 & 0.5552 & 0.0022 \\
\hline
\end{tabular}

\section{Conclusions}

Reanalysis methods are very useful to reduce the computational effort within applications, which are concerned with multiple design modifications, such as structural optimization, reliability analysis or damage analysis. In this contribution, a goal-oriented reanalysis method for the prediction of the change in a quantity of interest due to structural modifications by using the changes in the primal and dual solutions has been presented.

The changes in the primal and dual solutions due to given design modifications are computed by using the residual increment approximation (RIA) method. This reanalysis procedure is based on a formulation in terms of residual increments. In contrast to other existing reanalysis methods (e.g. the CA method), which are based on the evaluation of changed stiffness matrices, only residual vectors have to be computed and stored. The proposed method is very general and can be used for different kind of design modifications. The overall goal-oriented reanalysis procedure is straightforward and can easily be implemented in existing finite element programs, because no derivatives with respect to the design variables are necessary. The numerical examples presented in the paper demonstrate that the method yields very accurate results even for large design changes.

\section{REFERENCES}

[1] Oded Amir, Ole Sigmund, Boyan S. Lazarov, and Mattias Schevenels. Efficient reanalysis techniques for robust topology optimization. Comput. Methods Appl. Mech. Engrg., 245-246:217-231, 2012.

[2] Jasbir S. Arora. Survey of Structural Reanalysis Techniques. Journal of Structural Division (ASCE), 102:783-802, 1976.

[3] Franz-Joseph Barthold, Nikolai Gerzen, Wojciech Kijanski, and Daniel Materna. Mathematical 
Modeling And Optimization of Complex Structures, chapter Efficient variational design sensitivity analysis, pages 229-257. Springer-Verlag, 2016.

[4] K. K. Choi and N.-H. Kim. Structural Sensitivity Analysis and Optimization 1 - Linear Systems. Mechanical Engineering Series. Springer-Verlag, Berlin, 2005.

[5] Friedel Hartmann and Peter Jahn. Statics and Influence Functions - from a Modern Perspective. Springer International Publishing, 2017.

[6] Jan Holnicki-Szulc and Jacek T. Gierlinski. Structural modifications simulated by virtual distortions. Int. J. Numer. Meth. Engng, 28:645-666, 1989.

[7] Guanxin Huang, HuWang, and Guangyao Li. A reanalysis method for local modification and the application in large-scale problems. Struct Multidisc Optim, 49:915-930, 2014.

[8] Uri Kirsch. Reduced basis approximations of structural displacements for optimal design. AIAA Journal, 29:1751-1758, 1991.

[9] Uri Kirsch. Efficient sensitivity analysis for structural optimization. Comput. Methods Appl. Mech. Engrg., 117:143-156, 1994.

[10] Uri Kirsch. Combined approximations - a general reanalysis approach for structural optimization. Struct Multidisc Optim, 20:97-106, 2000.

[11] D. Materna and F.-J. Barthold. On variational sensitivity analysis and configurational mechanics. Computational Mechanics, 41(5):661-681, 2008.

[12] Daniel Materna. Structural and Sensitivity Analysis for the Primal and Dual Problems in the Physical and Material Spaces. Shaker Verlag, Aachen, 2010.

[13] Daniel Materna and V. K. Kalpakides. Nonlinear reanalysis for structural modifications based on residual increment approximations. Computational Mechanics, 57(1):1-18, 2016.

[14] A. Sidi. Rational approximations from power series of vector-valued meromorphic functions. $J$. Approx. Theory, 77:89-111, 1994.

[15] A. Turan and A. Muğan. Structural and sensitivity reanalyses based on singular value decomposition. Struct Multidisc Optim, 48:327-337, 2013.

[16] Baisheng $\mathrm{Wu}$, Zhengguang $\mathrm{Li}$, and Shunhua Li. The implementation of a vector-valued rational approximate method in structural reanalysis problems. Comput. Methods Appl. Mech. Engrg., 192:1773-1784, 2003. 